\title{
Performance Evaluation of a New Gamma Imager for Small Animal SPECT Applications
}

\author{
E Lage, JJ Vaquero Senior Member IEEE, J Villena, A de Carlos, G Tapias, A Sisniega, M Desco
}

\begin{abstract}
In this work we characterized a recently developed gamma imager for small animal SPECT applications. The Hamamatsu C9177 is a mini-gamma camera that integrates the detector and all the electronics, including the acquisition system, in a compact and portable housing. The detector is based on a high resolution parallel hole collimator, a $\mathrm{CsI}(\mathrm{NaI})$ crystal array and a PS-PMT (flat panel type). The active field of view is $41.9 \times 41.9 \mathrm{~mm}^{2}$ and the assembly is optimized for 60 to $200 \mathrm{keV}$. The electronics in the housing includes the high voltage divider, an ASIC which converts the 64 anodes into Anger-like signals, ADCs which are fed with these signals and position and energy lookup tables which allow digital information for each detected photon to be obtained directly from the imager. In order to be able to obtain tomographic data and to improve the measurement protocols, we mounted the detector in a custom-built motorized gantry. We evaluated detector uniformity and energy resolution using a flood field image. Planar intrinsic spatial resolution and spatial linearity were assessed by stepping a capillary source across the detector surface and plotting the count profile for each individual crystal of the array. We additionally performed phantom studies to preliminarily characterize the tomographic performance. Energy resolution is $\mathbf{1 1 . 6 \%}$ (mean), sensitivity is $2.54 \mathrm{cps} / \mu \mathrm{Ci}$ and planar spatial resolution is $2.4 \mathrm{~mm}(\mathrm{CFOV}$ $20 \%$ energy window) when the source is placed on the detector surface.
\end{abstract}

\section{INTRODUCTION}

INGLE photon emission computed tomography (SPECT) is a $N_{\text {molecular imaging technology that was rapidly adapted to }}$ animal imaging at high resolution. As well as other molecular imaging techniques, SPECT is based on the tracer principle and it is capable of measuring biodistributions of very small concentrations. Tracers with long half life are relatively easy to obtain, and labeling of endogenous ligands is a common practice in nuclear medicine departments, making this technique very attractive as a tool for translational research [12].

In this work, we have characterized a recently developed small gamma imager, with the purpose of using it as the basic detector on a small-animal SPECT/CT imaging system.

Manuscript received November 15, 2007. This work was supported in part by the Spanish Ministerio de Educación y Ciencia under Grant No. TEC2004-07052-C02, Comunidad de Madrid Grant No. GR/SAL/024104, and the CDTEAM project of the CENIT program (Spanish Ministerio de Industria).

Eduardo Lage, Juan José Vaquero, José Villena, Álvaro de Carlos, Gustavo Tapias, Alejandro Sisniega and Manuel Desco are with the Unidad de Medicina y Cirugía Experimental, Hospital General Universitario Gregorio Marañon, Madrid, Spain. (Corresponding author phone: +34914 265 067, email: elage@mce.hggm.es).
The document is organized as follows; in Section II we provide an overview of imager configuration and its technical features. In Section III we describe the planar and tomographic performance measurements carried out and discuss the effect of typical correction procedures in the resulting data, providing experimental evidences. Section IV summarizes and discusses some open issues and future works.

\section{THE C9177 RADIATION IMAGER}

The Hamamatsu C9177 is a mini-gamma camera that integrates the detector and all the electronics in a compact and portable housing [3]. The system is in-factory calibrated, providing a ready-to-use device with reasonable features.

One of the most interesting characteristics of this system is that it is an alternative to avoid using complicated custom-built designs, for the prototyping and testing of small animal SPECT tomographs.

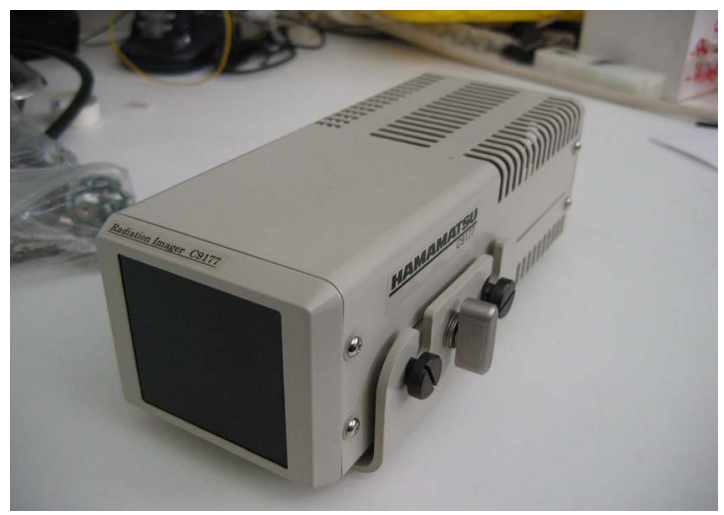

Figure 1. Front view of the Hamamatsu C9177 radiation imager. The system also includes a supply module, a connector box and a PCI interface. The approximate weight of the detector head is $2 \mathrm{Kg}$.

\section{A. Detector configuration}

The detector comprises the following components: a high resolution lead $(\mathrm{Pb})$ collimator with $1.2 \mathrm{~mm}$ hexagonal holes, $20 \mathrm{~mm}$ thick and $0.2 \mathrm{~mm}$ septal thickness; a $21 \times 21$ array of $1.9 \times 1.9 \times 6 \mathrm{~mm}^{3} \mathrm{CsI}(\mathrm{NaI})$ scintillator crystals ( $2 \mathrm{~mm}$ pitch), and a Hamamatsu R8400-M64 position sensitive photomultiplier tube, with an active area of $41.9 \times 41.9 \mathrm{~mm}^{2}$.

$$
\begin{aligned}
& R_{\text {coll }} \approx d\left(l_{\text {eff }}+b\right) / l_{e f f} \\
& g \approx K^{2}\left(d / l_{e f f}\right)^{2}\left[d^{2} /(d+t)^{2}\right]
\end{aligned}
$$

The performance of this configuration is strongly affected by both collimator geometrical efficiency $(g)$ and collimator resolution $\left(R_{\text {coll }}\right)$. The theoretical values for these parameters 
can be calculated using the approximated expressions (1-2), well described in [4]. The parameters $d$ and $b$ are respectively the diameter of the collimator holes and the distance from the radiation source to the collimator surface; $l_{\text {eff }}$ is the collimator's effective length which depends on the energy of the gamma rays to be detected, $t$ is the septal thickness between holes and $K$ is a constant that depends on the shape of the holes. Taking into account that the imager has been designed for work in the 60 to $200 \mathrm{keV}$ energy range, the theoretical collimator efficiency is roughly $2.71 \times 10^{-4}$ (photons transmitted/photons emitted). Also, in our small animal application, the maximum distance from the detector to the center of rotation is about 5 centimeters; therefore the expected values for $R_{\text {coll }}$ (with a $l_{\text {eff }}$ calculated for $150 \mathrm{keV}$ photons) vary from $1.2 \mathrm{~mm}$ on the surface of the camera to 4.3 $\mathrm{mm}$ at the center of rotation.

\section{B. Electronics and Data Interface}

The electronics in the housing integrates the high voltage supply and the divider circuit for the PMT dynodes. The main electronic component in the system is an ASIC which converts the value of the 64 anode outputs of the R8400 into three different output signals with information about the position and energy of the detected event. These outputs feed three analog-to-digital converters that integrate each signal for 4.2 $\mu$ s. The digitized values are then used as selection lines for dedicated ROMs, which maps each set of these signals with a single crystal and an energy channel. The system developers also included in these electronics a position and distortion correction algorithm.

The output interface of the detector head is based on a FIFO memory which stores the output value of the look-up tables until a readout order arrives from a control PC (Figure 2).

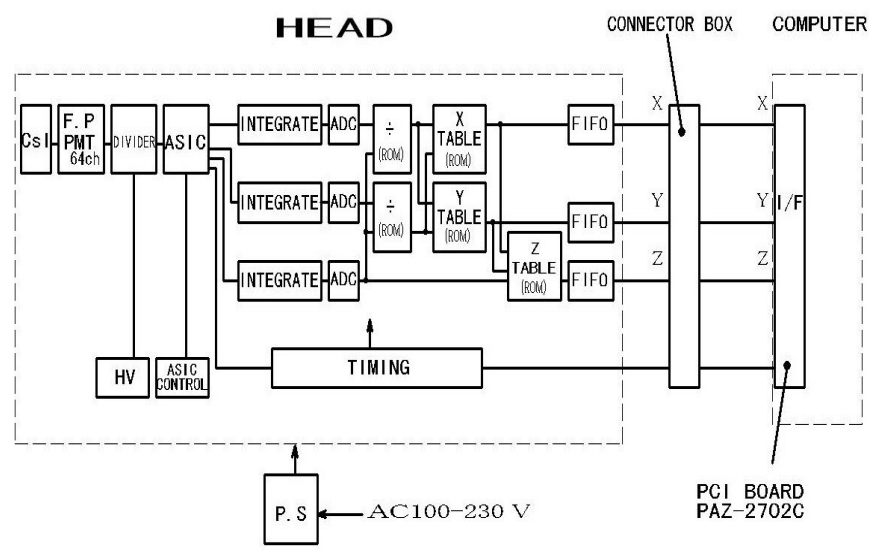

Figure 2. Internal functional diagram of the $\mathrm{C} 9177$ radiation imager. The electronic system is fed with the outputs of the PS-PMT and obtains digitized information of each detected event. This information is introduced in a lookup table system which converts the data from the ADCs to $\mathrm{X}$ and $\mathrm{Y}$ values with distortion and position corrected and an energy signal represented in the diagram as Z. The output interface of the head is comprised of a FIFO memory whose readout is triggered for an external interface.

The PAZ-270C (Interface Corp.) is a PCI card used as a circuit for supplying signals from the head to the computer. When the internal FIFO of the head becomes full (1024 events), a signal is sent to the PCI card. Transfer of data to the computer begins when this signal is received. When one item of data is captured, the computer sends another pulse to the head to prepare for the next data transfer. This signal has a pulse width which ranges from 1.5 to $4 \mu$ s and the time required to shift from one item to the next in the FIFO is 600 ns [3]. Each item of data from the detector head is 24 bits and is composed of three numbers which are 8 bits long. These represent the $\mathrm{X}, \mathrm{Y}$ position (scintillator position coordinates from 0 to 20), and the energy channel (0 to 254). When even one of the three data (X, Y, and Energy) is 255, it means that the item is invalid due to an error such as 2 radiation emissions being measured simultaneously.

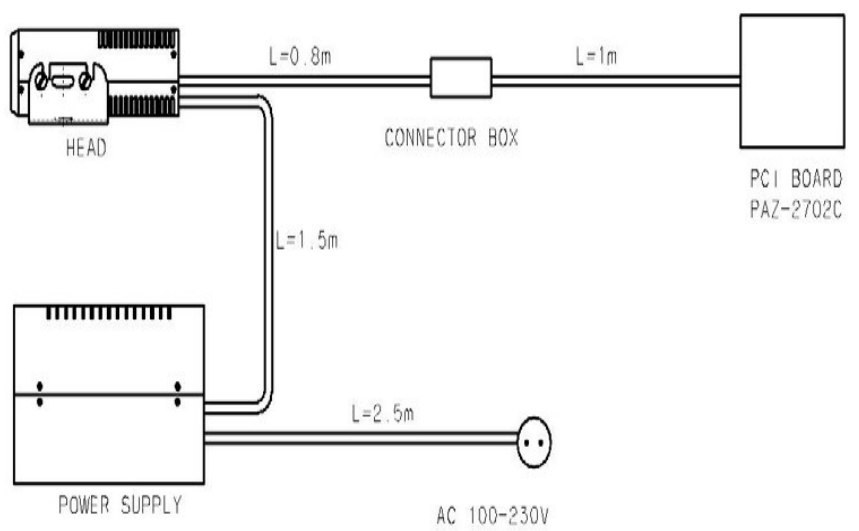

Figure 3. Connections and different modules that form the system. The power supply is used to set up the supply signals for the head. Inside the connector box, the signals are converted to match the PCI card and sent to the interface mounted inside the computer.

There are two additional modules included in the system; a box which provides the power supply to the detector head and a connector box which adequate the connectors between the head and the PCI board. A diagram of the whole system is shown in the Figure 3.

\section{PERFORMANCE MEASUREMENTS}

The C9177 is a non-versatile design with fixed features, i.e. after factory calibration, it's not possible acquire valid data without the collimator or with other different collimator. Due to this fact, it is not possible to evaluate all the system performance parameters using standard methods.

In order to take performance measurements with good precision and to acquire tomographic data, we started mounting the detector head on a motorized gantry as shown in the photographs of the Figure 4. Additionally, we programmed different motion and post-processing routines to carry out these measurements in a semi-automatic way and with high accuracy.

For the planar performance measurements, we evaluated both the detector features and the performance characteristics of the correction methods. For the tomographic measurements, we performed phantom studies using the Image Quality Phantom developed by the NEMA small-animal PET Standard Task Force and a home-made hot rods phantom. In these preliminary studies, slices were obtained using a Filtered Back-Projection algorithm. 

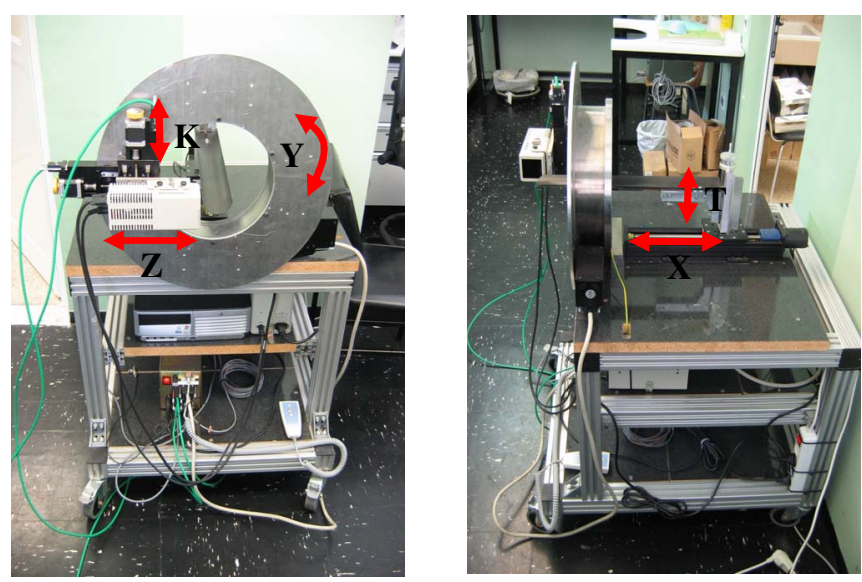

Figure 4. Gantry design for the small animal SPECT prototype. The system contains 4 motorized movement axes and a manual one. The $\mathrm{X}$ axis is for the bed and allows for whole body scans, the $\mathrm{Y}$ axis is a rotatory ring which allows the detector to be set in different angles. The $\mathrm{Z}$ axis allows the detector to be brought close to the object to imaging, the $\mathrm{K}$ axis allows for motions in the tangential direction and the $\mathrm{T}$ axis (the manual one) allows the sample to be raised and lowered. All the axes have submillimeter resolution.

\section{A. Differential and Integral Uniformity}

We evaluated the detector uniformity and the energy resolution using a field flood image of the detector. The flood source consisted of a plastic container filled with $2 \mathrm{mCi}$ of ${ }^{99 \mathrm{~m}} \mathrm{Tc}$ which was acquired for 12 hours. This phantom is sufficiently large to cover the entire detector and was placed in direct contact with the collimator.

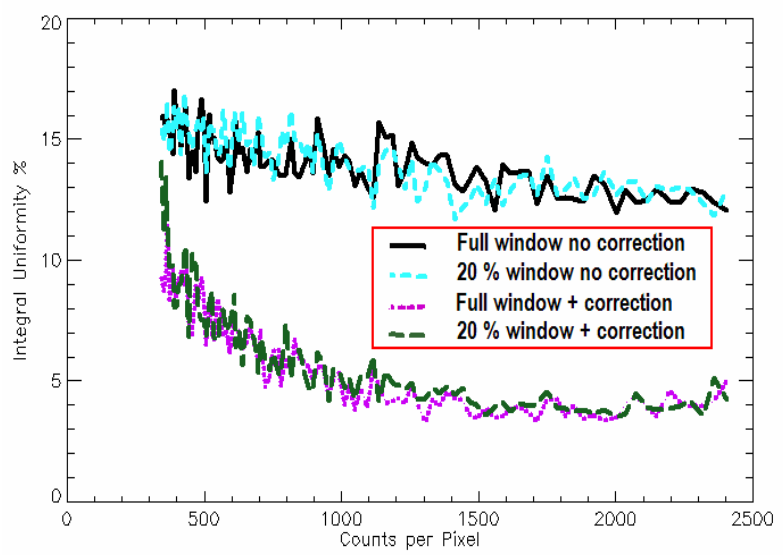

Figure 5. Integral uniformity of the detector as a function of the count density of the image to correct. The measurement was done over a centered FOV containing $80 \%$ of the total active area. The plots also show the effectiveness of the non-uniformity correction algorithm.

In order to improve the uniformity in the UFOV, we obtained two different correction files from the flood data. The first one was used as an energy map which contains offset values, necessary to align the energy spectrum of each crystal to a common reference. After the application of the latter correction file to the original flood data, we obtained a projection image using a common energy window and compared the number of counts recorded within each pixel with the mean pixel value, obtaining a non-uniformity correction file with the relative efficiency of each image element for the selected energy window.

The imager non-uniformity and stability were evaluated acquiring a new set of extrinsic flood field images using the same phantom than in the latter case and with a decaying number of counts. Integral uniformity [6] was calculated before and after applying the non-uniformity correction algorithm and for different energy windows. The resulting values for these measurements are shown in Figure 5 as a function of the count density in the non-corrected full energy window images.

Differential uniformity was calculated vertically and horizontally over the same images and after applying three different energy windows. The resulting values for the UFOV and CFOV in the "stable zone" (approximately more than 1000 counts per pixel in the image to correct, see Figure 5) are shown in Table 1.

TABLE I

INTEGRAL UNIFORMITY \%

\begin{tabular}{cccccc}
$\begin{array}{c}\text { Energy } \\
\text { Window }\end{array}$ & $\begin{array}{c}\text { Uniformity } \\
\text { Correction }\end{array}$ & $\begin{array}{c}\text { Horizontal } \\
\text { UFOV }\end{array}$ & $\begin{array}{c}\text { Vertical } \\
\text { UFOV }\end{array}$ & $\begin{array}{c}\text { Horizontal } \\
\text { CFOV }\end{array}$ & $\begin{array}{c}\text { Vertical } \\
\text { CFOV }\end{array}$ \\
\hline $100 \%$ & No & 16.248 & 8.746 & 8.092 & 5.710 \\
$20 \%$ & No & 7.338 & 11.03 & 7.312 & 7.231 \\
$10 \%$ & No & 11.34 & 12.27 & 6.950 & 8.305 \\
$100 \%$ & Yes & 1.861 & 1.934 & 0.884 & 1.200 \\
$20 \%$ & Yes & 2.276 & 1.994 & 0.955 & 1.138 \\
$10 \%$ & Yes & 2.755 & 2.241 & 1.025 & 1.267 \\
\hline
\end{tabular}

\section{B. Energy Resolution}

As explained in the previous section, in the first step of the correction procedure, we aligned the energy spectrum of each crystal to a common reference. During this process, we fitted a portion of the energy spectrum of each pixel to a Gaussian function and obtained the photopeak position for each image element. The offset value was calculated as the difference between the average photopeak channel (average of the position of all the Gaussian function centers) and the photopeak position for each crystal. For the C9177 imager, and in this experiment, it was really a fine tuning process because of the fact that the photopeak channel position varied on average less than $2 \%$ over the calculated reference.

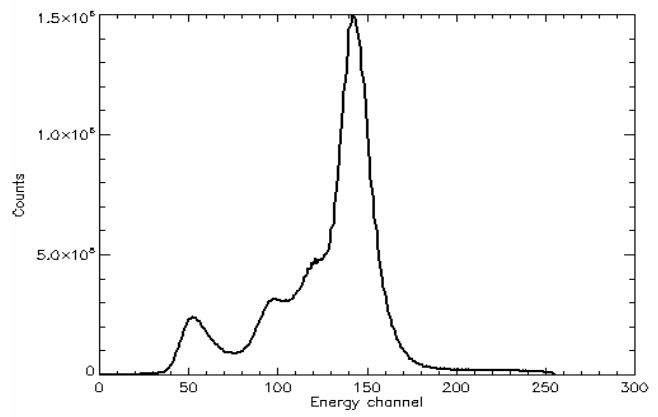

Figure 6. Energy spectrum for the entire detector after non-uniformity correction for a ${ }^{99 \mathrm{~m}} \mathrm{Tc}$ flood source. Energy resolution expressed as a percentage of the ${ }^{99 \mathrm{~m}} \mathrm{Tc}$ photopeak energy is $11.6 \%$. 
The Figure 6 shows the energy spectrum of the entire detector after align the energy spectrum of each pixel and the application of the non-uniformity correction file. Energy resolution measured across the detector and expressed as a percentage of the ${ }^{99 \mathrm{~m}} \mathrm{Tc}$ photopeak energy is $11.6 \%$ average, $20.41 \%$ for the worst crystal and $8.99 \%$ for the best one. The camera specifications indicate a $20 \%$ energy resolution value, measured intrinsically and with a ${ }^{57} \mathrm{Co}$ source.

\section{Planar Spatial Resolution}

In order to measure the intrinsic spatial resolution, we stepped a capillary source across the detector surface and plotted the count profile for each individual crystal of the array. With the purpose of perform this process with sufficient accuracy, we implemented a software routine to acquire projections automatically in different positions of the detector (for this study, movements was done in the $\mathrm{K}$ and $\mathrm{X}$ axis, see Figure 4).

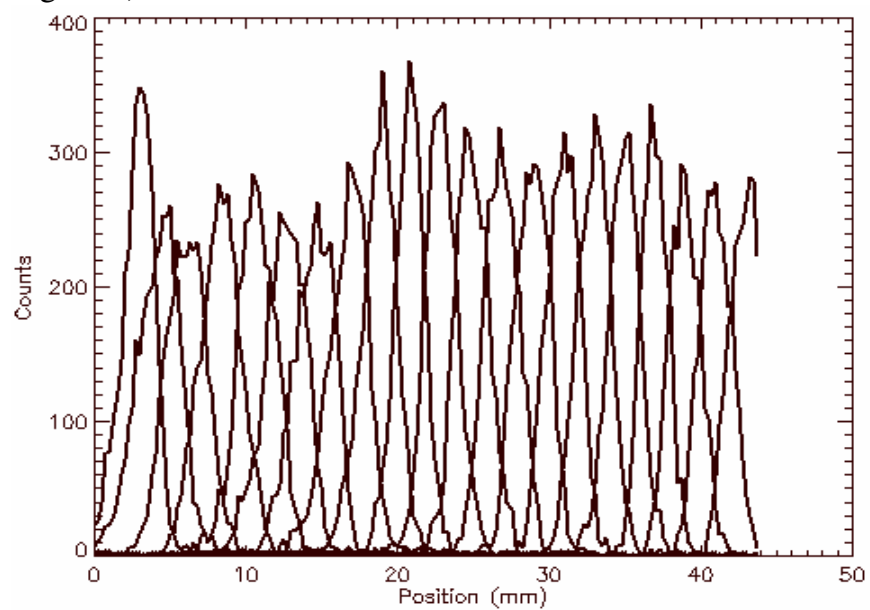

Figure 7. Count profiles for each individual crystal in column 10 of the detector. In these measurements, we fitted each count profile to a Gaussian function. The spatial resolution was computed as the mean FWHM for all the pixels of the detector in the $\mathrm{x}$ and $\mathrm{y}$ direction. Spatial linearity was computed using the distance between consecutive maximum values in the profiles.

Before starting the acquisition we aligned the capillary source $(1 \mathrm{~mm}$ internal diameter and $7 \mathrm{~cm}$ length) with the bottom edge of the camera surface. The capillary was filled with $500 \mu \mathrm{Ci}$ of ${ }^{99 \mathrm{~m}} \mathrm{Tc}$ and the acquisition was in $0.25 \mathrm{~mm}$ steps from the bottom to the upper edge of the detector, acquiring a 60 seconds projection for position. The same experiment was performed moving the source from left to right across the entire FOV, stepping in this case in the $\mathrm{X}$ direction (see Figure 4). After correcting decay and non-uniformity of the projections, we plotted the count profile for each individual crystal of the array vertically and horizontally. These profiles were fitted to Gaussian functions and the average system FWHM was calculated as the mean value for the single results in the horizontal and vertical scans.

In terms of the FWHM of a PSF or LSF, the most important factors are the intrinsic resolution $\left(R_{\text {int }}\right)$ of the detector and the electronics, and the collimator resolution $\left(R_{\text {coll }}\right)$. The combined effect of these two factors is to produce a system resolution $\left(R_{\text {sys }}\right)$ given by (3).

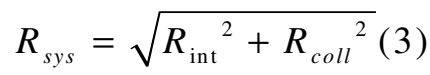

The results for this measure are summarized in table 2; the columns 3 and 4 show the average FWHM for the entire detector in the $\mathrm{X}$ and $\mathrm{Y}$ directions. The mean $R_{s y s}$ column is the mean value for the two previous and the $R_{\text {int }}$ value of the detector was obtained using the mean $R_{s y s}$ values in formula (3). The resulting system resolution is $2.4 \mathrm{~mm}$ for the $20 \%$ energy window measured over the CFOV. The mean values for the entire detector over the UFOV are shown in Table II for different imaging conditions.

TABLE II

SPATIAL RESOLUTION (MILIMITERS)

\begin{tabular}{cccccc}
$\begin{array}{c}\text { Energy } \\
\text { Window }\end{array}$ & $\begin{array}{c}\text { Uniformity } \\
\text { Correction }\end{array}$ & $\begin{array}{c}\text { Horizontal } \\
\mathrm{R}_{\text {sys }}\end{array}$ & $\begin{array}{c}\text { Vertical } \\
\mathrm{R}_{\text {sys }}\end{array}$ & $\begin{array}{c}\text { Mean } \\
\mathrm{R}_{\text {sys }}\end{array}$ & $\begin{array}{c}\text { Mean } \\
\mathrm{R}_{\text {int }}\end{array}$ \\
\hline $100 \%$ & No & 2.949 & 2.870 & 2.917 & 2.659 \\
$20 \%$ & No & 2.835 & 2.759 & 2.797 & 2.527 \\
$10 \%$ & No & 2.727 & 2.692 & 2.709 & 2.429 \\
$100 \%$ & Yes & 2.750 & 2.710 & 2.750 & 2.475 \\
$20 \%$ & Yes & 2.676 & 2.610 & 2.643 & 2.355 \\
$10 \%$ & Yes & 2.597 & 2.574 & 2.585 & 2.290 \\
\hline
\end{tabular}

Resolution was also plotted as a function of distance between the detector and the source position (Figure 8). This experiment shows the effect of the parallel-hole collimator resolution and is in good agreement with the literature.

In this measurement we acquired the same capillary source filled with $430 \mu \mathrm{Ci}$ of ${ }^{99 \mathrm{~m}} \mathrm{Tc}$, first aligned with the central row and second aligned with the central column of the crystal array. During the acquisitions, we varied the source-tocollimator distance by stepping the detector in the Z-axis direction (Figure 4) with $2.5 \mathrm{~mm}$ steps and acquiring a 60 seconds projection in each position. After correcting the decay of the projections, we calculated the LSF in each image in different conditions and plotted the system resolution versus the source-to-collimator distance.

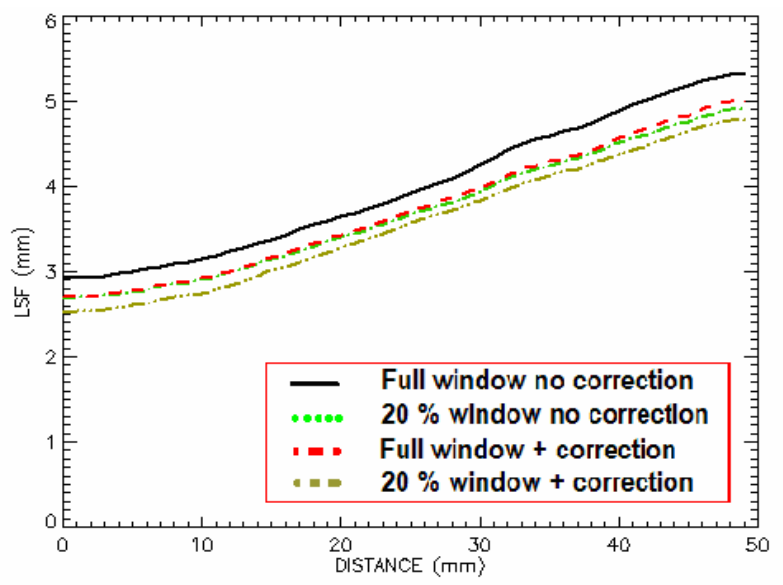

Figure 8. Horizontal system resolution versus source to collimator distance. The capillary source was aligned with the central line off the detector and stepped in the Z-axis varying the source-to-collimator distance. The "correction" word in the chart's legend indicates that nonuniformity correction was applied to the data before plot. 


\section{Spatial linearity}

To evaluate the spatial linearity of the detector, we used the data of the previous experiment (Figure 7) and fitted then with a set of displaced Gaussian functions. We obtained the differential and absolute spatial linearity by computing the distance between consecutive maximum values (centers of the Gaussian functions) for each row and column as well as its difference with their theoretical position. Results for differential and absolute spatial linearity calculations are summarized in Table III. CFOV and UFOV Average refers to that the value shown in the table is the average value for the vertical and horizontal scans.

TABLE III

SPATIAL LINEARITY (MILIMITERS)

\begin{tabular}{ccc} 
& $\begin{array}{c}\text { CFOV } \\
\text { Average }\end{array}$ & $\begin{array}{c}\text { UFOV } \\
\text { Average }\end{array}$ \\
\hline Mean & 0.2939 & 0.1791 \\
Std. Dev. & 0.3072 & 0.2708 \\
\hline
\end{tabular}

\section{E. System sensitivity}

System sensitivity was measured using a $3 \mathrm{~cm}$ diameter, 3 $\mathrm{mm}$ deep dish, filled with $2 \mathrm{mCi}$ of ${ }^{99 \mathrm{~m}} \mathrm{Tc}$ which was placed at $10 \mathrm{~cm}$ from the face of the collimator. After acquiring an image for 20 minutes, we drew a circular region of interest and integrated all the counts in that region.

$$
\text { Sens }=\frac{\text { Counts_ROI }_{-} \text {Background_ROI }}{\text { Time }(s) x \text { Activity }(u C i)}(4)
$$

A second image was acquired for an equal time with the source removed, to provide a measure of the background. The same region of interest was applied to this image, and the sensitiviy value was obtained in $\mathrm{cps} / \mu \mathrm{Ci}$ using (4) and obtaining a value of $2.54 \mathrm{cps} / \mu \mathrm{Ci}$. For the calculations, images are with a $100 \%$ energy window and non-uniformity correction was not applied.

\section{F. Tomographic Performance}

Since the results in $2 \mathrm{D}$ have shown good resolution capability, tomographic studies have also been performed in order to evaluate the possibility of use of the C9177 imager in a small-animal SPECT/CT system.

The Contrast to Noise Ratio (CNR) was measured using a NEMA approved phantom for image quality assessment [6]. This phantom is mainly composed of three different containers (Figure 9 left) which were filled with ${ }^{99 \mathrm{~m}} \mathrm{Tc}$ and with different activity levels (H: Hot $1.3 \mathrm{mCi} / \mathrm{cc}, \mathrm{C}$ : Cold $0 \mathrm{mCi} / \mathrm{cc}, \mathrm{B}$ : Background $0.33 \mathrm{mCi} / \mathrm{cc})$. After image reconstruction, we segmented in the $3 \mathrm{D}$ volume the $\mathrm{B}, \mathrm{H}$ and $\mathrm{C}$ regions, and obtained the mean count value and the standard deviation for each ROI. The CNR in the image was calculated using (5) obtaining a $3.7 \%$ value.

$$
C N R=\frac{\frac{\left(\mu_{H}-\mu_{B}\right)}{\mu_{B}}}{\sqrt{\sigma_{H}^{2}+\sigma_{B}^{2}}}
$$

Spatial resolution was calculated using the background slices of the phantom (Figure 9 right). In this case, we obtained the detector Edge Response Function (ERF) drawing various profiles in the edge of the phantom and averaging then. The derivate of the ERF is the Line Spread Function (LSF) of the detector. The FWHM of the LSF obtained in this way (30\% Energy window, FBP + ramp filter reconstruction) was of 3.6 $\mathrm{mm}$.
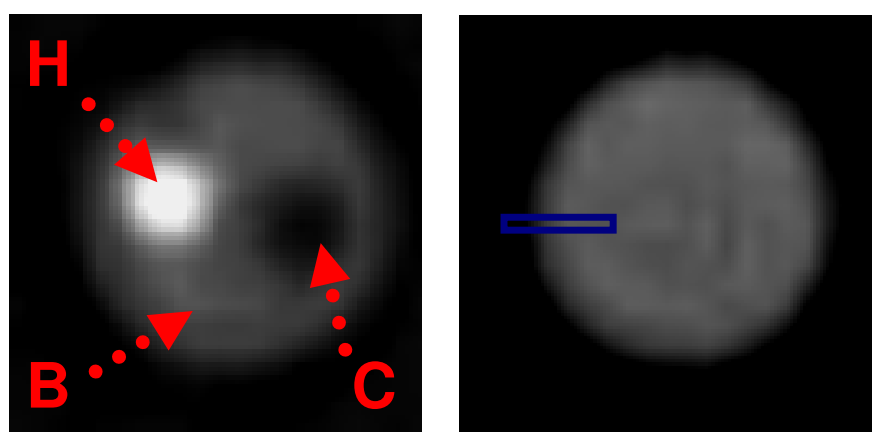

Figure 9. Two reconstructed slices of the NEMA phantom. The left image shows a zone with 3 different activity levels (C: Cold, $\mathrm{H}$ : Hot and $\mathrm{B}$ : Background). Te right image shows a zone with uniform activity level which was used to calculate the spatial resolution.

In a second experiment and in order to ensure the spatial linearity previously measured in planar images, we performed a study of a home-made phantom containing capillary sources of 6, 4.8 and $3 \mathrm{~mm}$ inner diameters, placed at different intercapillary distances (Figure 10). We traced profiles in the reconstructed slices and measured the correspondence of the imaged distances with the real ones obtaining a good agreement with the planar spatial linearity measurements.

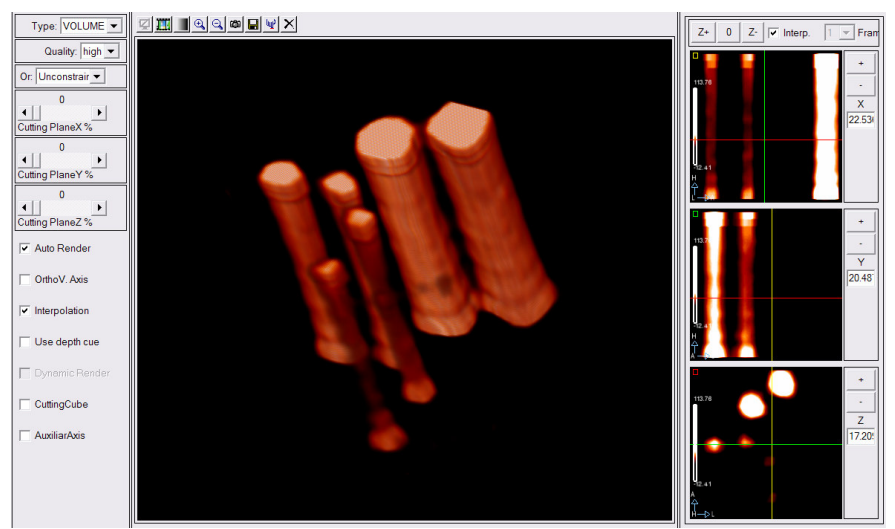

Figure 10. Volumetric render of a home-made phantom containing capillary sources of diameters $(6,4.8$ and $3 \mathrm{~mm})$. Spatial linearity was assessed obtaining well correspondence of the imaged distances with the real ones. Slices were reconstructed using an FBP algorithm and a Ramp Filter.

\section{Discuss AND CONCLUSIONS}

We have characterized the planar performance features of the C9177 radiation imager. The effect of the non-uniformity correction method in spatial resolution of the system was also evaluated by assessing the data before and after the corrections.

The achieved uniformity values are comparable to values of clinical grade cameras, which usually are $<5 \%$ uniformity for 
acceptable human imaging. The plot of Figure 5, indicates that with only a detector and at typical activity levels in small animal SPECT, the number of counts required to obtain acceptable non-uniformity values is roughly $275 \times 10^{3}$, which is equivalent to approximately 950 counts per pixel in the image to correct. Results in 3D studies show good contrast and spatial linearity as well as acceptable spatial resolution although it can be further improved using improved acquisition routines and reconstruction algorithms.

We have developed a demonstrator prototype of SPECT tomograph based on the C9177 imager; the performance characteristics of this unit and its compact enclosure make it suitable for certain small animal imaging applications. However, in our opinion the imager design still presents certain problems which difficult its use in a multi-detector scanner, i.e. the use of a PCI interface for read-out the data or its configuration non-versatility.

\section{ACKNOWLEDGMENT}

We thank Hamamatsu Photonics and Dr. D. Castrillo from Hamamatsu Spain for his support. We thank Dr. P. Dominguez from the department of Nuclear Medicine of the Gregorio Marañón Hospital for his support with the measurements.

\section{REFERENCES}

[1] FD Rollo. Nuclear Medicine physics, instrumentation and agents, First edition, The C,V. Mosby Company. ISBN0-8016-4181-0.

[2] S.R. Meiklel, P Kench, M Kassiou. "Small Animal SPECT and its place in matrix of molecular imaging technologies", Phys. Med. Biol. 50 (2005) R45-R61

[3] HAMAMATSU Photonics K.K. Radiation image Measurements device c9177 mod instruction Manual, 2007.

[4] SR Cherry, JA Sorenson, ME Phelps. Physics in Nuclear Medicine, third edition Elsevier Science (USA), ISBN 0-7216-8341-X 2003

[5] JESRA Standard, "http://www rada.or.jp/database/index.html"

[6] NEMA Standard, http://www.nema.org/stds/ 